\title{
Unraveling the molecular mechanisms of hyperlipidemia using integrated IncRNA and mRNA microarray data
}

\author{
BIANLING XU ${ }^{1-3^{*}}$, NAN WANG ${ }^{2 *}$, XUEGONG XU ${ }^{2,3}$ and YONGMIN CAI ${ }^{1,4}$ \\ ${ }^{1}$ Institute of Chinese Medicine Literature, Nanjing University of Chinese Medicine, Nanjing, Jiangsu 210023; \\ ${ }^{2}$ Laboratory of Zhengzhou Hospital of Traditional Chinese Medicine, Zhengzhou, Henan 450007; \\ ${ }^{3}$ Department of Internal Medicine of Traditional Chinese Medicine, The First Clinical Medicine Department; \\ ${ }^{4}$ Zhang Zhongjing Inheritance and Innovation Center, Henan University of Chinese Medicine, \\ Zhengzhou, Henan 450046, P.R. China
}

Received August 13, 2020; Accepted June 9, 2021

DOI: 10.3892/etm.2021.11083

\begin{abstract}
Long non-coding RNAs (lncRNAs) have key roles in various diseases; however, their functions in hyperlipidemia (HLP) have remained elusive. In the present study, microarray technology was utilized to analyze the differential expression of lncRNAs and mRNAs in liver tissues of apolipoprotein $\mathrm{E}^{-/-}$ mice as a model of HLP compared with control mice. A total of 104 and 96 differentially expressed lncRNAs and mRNAs, respectively, were identified. Differentially expressed genes were significantly enriched in biological processes such as nitric oxide biosynthesis, innate immune response and inflammatory response. Finally, two pairs of target genes and 38 transcription factors with regulatory functions in HLP were predicted based on the lncRNA and mRNA co-expression network. The lncRNA expression profile was significantly altered in liver tissues of the mouse model of HLP and may provide novel targets for research into treatments.
\end{abstract}

\section{Introduction}

Hyperlipidemia (HLP) is a common and frequently encountered metabolic disease characterized by abnormally elevated total cholesterol (TC) and/or triglyceride (TG) levels in the plasma, resulting in dormant, progressive and serious damage to various organs, particularly the liver (1). The liver is the major site for intermediary metabolism and the major organ for the synthesis and metabolism of endogenous blood lipids and lipoproteins. Extrahepatic cholesterol is mainly carried

Correspondence to: Professor Yongmin Cai, Institute of Chinese Medicine Literature, Nanjing University of Chinese Medicine, 138 Xianlin Avenue, Nanjing, Jiangsu 210023, P.R. China

E-mail: caiym0629@163.com

${ }^{*}$ Contributed equally

Key words: hyperlipidemia, ApoE ${ }^{-/-}$mice, lncRNAs, differentially expressed genes, co-expression network by high-density lipoprotein (HDL) and transported to the liver via the HDL receptor for metabolism (2). Infiltration of liver tissues by lipids, including TG, leads to hepatic degeneration and may cause dysregulation of lipid metabolism and lipoprotein synthesis, particularly reduced HDL synthesis and defective synthesis of very low-density lipoprotein (VLDL) in the liver (3). As a result, hepatic lipids cannot be exported via lipoproteins, leading to the accumulation of TC and TG in the liver, ultimately resulting in hepatic insufficiency and dysregulation of lipid metabolism (4). Eventually, over-accumulation of lipids caused by dysregulated lipid metabolism results in HLP. Despite the progress in elucidating the pathogenesis of HLP, the underlying molecular mechanisms have remained to be fully clarified.

Although $98 \%$ of the DNA sequences of the human genome have been transcribed, protein-coding genes account for $<2 \%$ of the genome. The remaining genomic sequences are transcribed into non-coding RNAs (ncRNAs) (5). Based on their length, ncRNAs are categorized into two classes: ncRNAs smaller than 200 bp are usually referred to as small ncRNAs, including small interfering RNA, microRNA and piWi-interacting RNA, whereas long ncRNAs (lncRNAs) are defined as transcripts longer than 200 bp (6). lncRNAs have important roles in various critical biological processes at the transcription level, including genomic imprinting, chromatin inactivation, differentiation and carcinogenesis (7). Furthermore, accumulating evidence indicates that lncRNAs are closely related to the development and progression of cardiovascular and myocardial infarction $(8,9)$. For instance, lncRNAs are involved in the adipogenesis of white adipose tissue, energy metabolism of brown adipocytes (10) and accumulation of lipid droplets (11). Furthermore, myosin heavy-chain-associated RNA, an IncRNA abundant in adult hearts, has been suggested to protect the heart from cardiac hypertrophy and heart failure (12). However, the expression profile and biological function of lncRNAs in HLP remain elusive.

As a ligand, apolipoprotein E (ApoE) is able to contribute to the clearance of chylomicrons and VLDL and the lack of ApoE may lead to lipoprotein metabolism disorder and predispose an individual to lipoprotein deposition in the arterial 
wall. The pathological development of atherosclerosis in ApoE knockout mice is highly similar to that in humans and ApoE knockout mice are prone to developing severe hyperlipidemia.

Therefore, in the present study, a microarray analysis of the liver tissue of a model of HLP using ApoE knockout (ApoE ${ }^{-/}$) mice and from control mice was performed using the Mouse IncRNA Array v1.0, 4x180K (CapitalBio Technology, Inc.,) to identify differentially expressed lncRNAs and mRNAs. Bioinformatics tools were used to functionally annotate the differentially expressed genes (DEGs) and identify related transcripts. Collectively, these results provide novel insight into the molecular mechanisms of HLP and suggest novel therapeutic targets for future research.

\section{Materials and methods}

Animal model. A total of 12 male 6-week-old C57BL/6J mice (weight, 23.0 $\pm 0.74 \mathrm{~g}$ ) and 12 male $\mathrm{ApoE}^{-/-}(\mathrm{C} 57 \mathrm{BL} / 6 \mathrm{~J})$ mice (weight, $23.0 \pm 0.57 \mathrm{~g}$ ) were purchased from Beijing Vital River Laboratory Animal Technology. After 7 days of acclimation to the specific pathogen-free environment at Henan University of Chinese Medicine Laboratory Animal Center (Zhengzhou, China), the animals were assigned to two groups, namely a Normal control group $(n=12)$ and an HLP group $(n=12)$. The normal control group consisted of wild-type C57BL/6J mice with a regular mouse diet $(65.08 \%$ carbohydrates, $11.85 \%$ fat, and $23.07 \%$ protein). In the HLP group six-week-old male $\mathrm{ApoE}^{-/-}$mice were fed with a high-fat diet (HFD) containing $2.5 \%$ cholesterol and $15 \%$ fat. HFD was purchased from Beijing HFK Bioscience. The animals were housed in a temperature-controlled room $\left(22-25^{\circ} \mathrm{C}, 45 \%\right.$ humidity) with a 12-h light/dark cycle for 16 weeks. Animal behavior and health were monitored every day. On the day tissue and blood collection were to be performed, mice were fasted beginning at 9 am for a period of $5 \mathrm{~h}$ and blood collection procedures were initiated at $2 \mathrm{pm}$. A total of 600-800 $\mu \mathrm{l}$ blood was collected from each mouse. Blood from all the 24 mice was collected via retro-orbital bleeding under anesthesia by intraperitoneal injection of sodium pentobarbital (50 mg/kg body weight). The anesthesia state of the mice was observed; if the mice had movement or pain, a supplementary dose of $50 \mathrm{mg} / \mathrm{kg}$ (to reach $100 \mathrm{mg} / \mathrm{kg}$ in total) was added (13). After blood collection, the 24 mice were euthanized with an overdose $(150 \mathrm{mg} / \mathrm{kg}$ in total $)$ of pentobarbital. A combination of criteria was most reliable in confirming death, including lack of pulse, breathing, corneal reflex and response to firm toe pinch, inability to hear respiratory sounds and heartbeat by use of a stethoscope, graying of the mucous membranes and rigor mortis (14). Once euthanasia was performed, the liver tissues of the mice were immediately collected and stored in liquid nitrogen for further analysis. All of the procedures followed the requirements of the Ethics Committee of Henan University of Chinese Medicine (ethics review approval code no. DWLL201704101).

Blood lipid measurement and $H \& E$ staining. Blood was collected from mice fasted from 9 am to $2 \mathrm{pm}$ and the serum was separated by centrifugation at $5,000 \mathrm{x}$ g for $5 \mathrm{~min}$ at $22 \pm 2^{\circ} \mathrm{C}$. The mice were anesthetized for the collection of blood and liver samples. All samples were stored in liquid nitrogen for the subsequent analyses. An automatic biochemistry analyzer
(Siemens AG) was used for quantifying lipid concentrations, including TG, TC and LDL cholesterol (LDL-C) in the serum. Formalin-fixed liver tissues were embedded in paraffin and sections $(4 \mu \mathrm{m})$ were cut and stained with H\&E (Biyuntian).

RNA extraction, labeling and hybridization. Total RNA was extracted from homogenized liver tissues using TRIzol ${ }^{\circledR}$ reagent (Invitrogen; Thermo Fisher Scientific, Inc.) according to the manufacturer's protocol. cDNA labeled with a fluorescent dye (cyanine 3-dCTP) was produced by Eberwine's linear RNA amplification method and subsequent enzymatic reaction. Double-stranded cDNAs (containing the T7 RNA polymerase promoter sequence) were synthesized from $1 \mu \mathrm{g}$ total RNA using the CbcScript reverse transcriptase with cDNA synthesis system according to the manufacturer's protocol (CapitalBio Technology, Inc.) with the T7 Oligo(dT) and T7 Oligo(dN). After completing double-stranded cDNA (dsDNA) synthesis using DNA polymerase and RNase H (MACHEREY-NAGEL $\mathrm{GmbH} \& \mathrm{Co} . \mathrm{KG}$ ), the dsDNA products were purified using a PCR NucleoSpin Extract II Kit (MACHEREY-NAGEL GmbH \& Co. KG). $14 \mu$ l DNA was denatured in hybridization solution (Agilent Technologies, Inc.) at $95^{\circ} \mathrm{C}$ for $3 \mathrm{~min}$ prior to loading onto a microarray (Mouse lncRNA Array v1.0, 4x180K; CapitalBio Technology, Inc.). Arrays were hybridized in a hybridization oven (Agilent Technologies, Inc.) overnight at a rotation speed of $20 \mathrm{rpm}$ and a temperature of $42^{\circ} \mathrm{C}$ and washed with two consecutive solutions $(0.2 \%$ SDS, 2 X SSC at $42^{\circ} \mathrm{C}$ for $5 \mathrm{~min}$ and $0.2 \mathrm{X} \mathrm{SSC}$ at $23-26^{\circ} \mathrm{C}$ for $5 \mathrm{~min}$ ).

Microarray imaging and data analysis. GeneSpring v13.0 software (Agilent Technologies, Inc.) was used to analyze lncRNA and mRNA microarray data and perform summarization, normalization and quality control. To identify DEGs, the threshold for the fold-change was set at $\geq 2$ or $\leq-2$ and a Benjamini-Hochberg-adjusted P-value of 0.05 was set as the cut-off for statistically significant results. $\log _{2}$ transformation of the data was performed using CLUSTER 3.0 software (http://bonsai.hgc.jp/ mdehoon/software/cluster/software.htm) and the genes were centered, followed by average-linkage hierarchical clustering analysis. The data were visualized using Java Treeview 1.1.6 (Stanford University School of Medicine).

Functional group analysis. Gene Ontology (GO) analysis was performed to explore the functions of DEGs identified in the present study. The Database for Visualization and Integrated Discovery (DAVID) online tool (https://david.ncifcrf.gov/) was utilized to perform functional enrichment analysis of DEGs, which classified, identified and annotated the genes in terms of the GO categories of biological process, cellular component and molecular function. The P-values were adjusted using the false discovery rate and the threshold for significant differences was set at $\mathrm{P}<0.05$.

Coding-non-coding gene co-expression network analysis. The coding-non-coding gene co-expression network was constructed based on the correlation analysis among differentially expressed lncRNAs and mRNAs. For each pair of genes, the Pearson's correlation coefficient was determined and pairs with a significant correlation were selected to construct the network. The lncRNAs and mRNAs with Pearson's correlation 
A

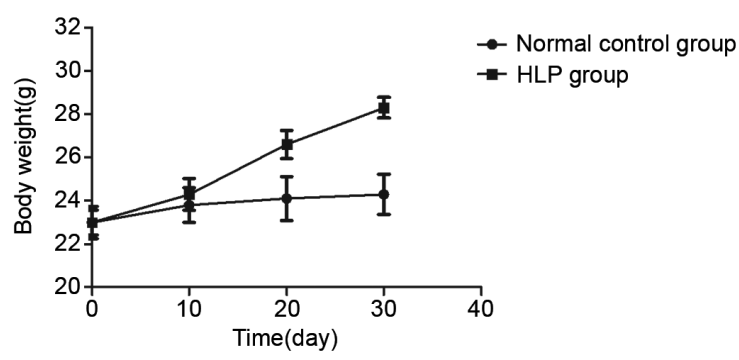

C

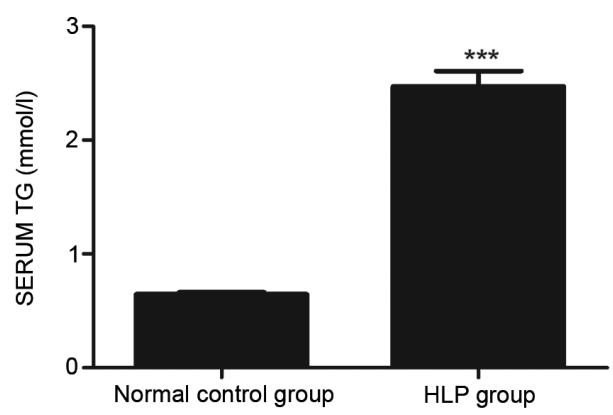

B

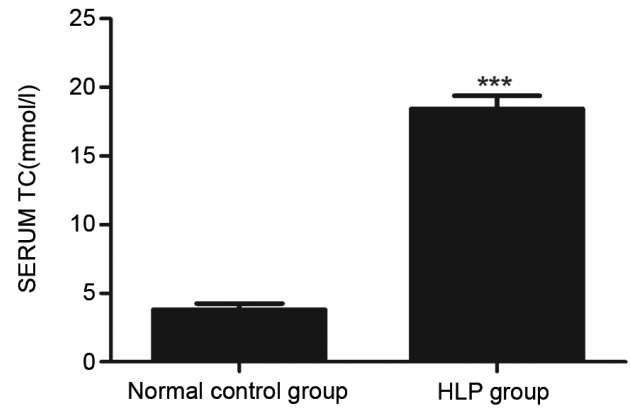

$\mathrm{D}$

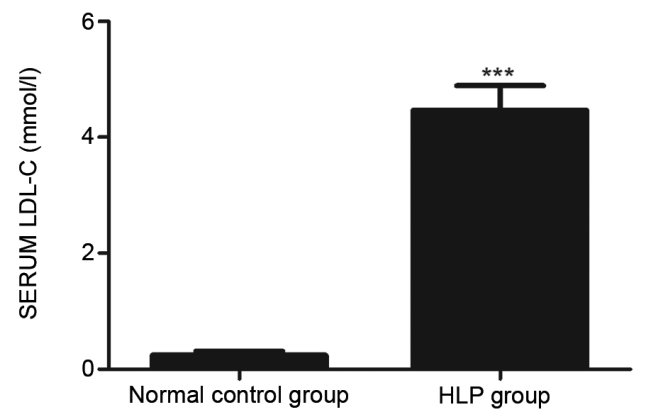

Figure 1. Bodyweight and serum lipid changes of mice of the HLP group and Normal control group. (A) Bodyweight, (B) TC, (C) TG and (D) LDL-C. Mice from the HLP group were apolipoprotein $\mathrm{E}^{--}$and were fed a high-fat diet and the normal control mice were fed a regular diet for 16 weeks. ${ }^{* * *} \mathrm{P}<0.001 \mathrm{vs}$. Normal control group. HLP, hyperlipidemia; TC, total cholesterol; TG, triglyceride; LDL-C, low-density lipoprotein cholesterol.

coefficients of no $<0.99$ were incorporated into the network using the bioinformatics software Cytoscape 3.6.1 (https:// cytoscape.org/download.html).

Prediction of target genes. The cis-acting lncRNA prediction of a group of expressed protein-coding genes was performed based on the close association of lncRNA-mRNA pairs (minimum Pearson's correlation coefficient of 0.99). Trans-prediction was performed using BLAT tools (Standalone BLAT v.35x1 fast sequence search command line tool downloaded from http:// hgdownload.cse.ucsc.edu/admin/exe/) to compare the full sequence of an IncRNA with the 3 ' untranslated region of its co-expressed mRNA using default parameter settings.

Prediction of transcription factors. The transcription factor prediction database TFactS was downloaded from http://www. tfacts.org. Based on the co-expression data of differentially expressed lncRNAs and mRNAs, the differentially expressed mRNAs were compared with those in the database and the correlations of co-expressed lncRNAs and mRNAs with the transcription factors were obtained. The regulatory network diagrams of co-expressed lncRNAs and mRNAs with transcription factors were drawn using Cytoscape 3.6.1.

Statistical analysis. Statistical analyses were performed using GraphPad Prism 5.0 Software (GraphPad Software, Inc.). Data were analyzed for differences between the Normal control group and the HLP group using an unpaired Student's t-test. Correlation analysis was performed using Pearson's correlation analysis. $\mathrm{P}<0.05$ was considered to indicate a statistically significant difference.

\section{Results}

Bodyweight and serum lipid level variations in the HLP model. Fig. 1A presents the measured bodyweights of mice in the Normal control group and HLP groups at days 10, 20 and 30 during continuous feeding of the regular and high-fat diet, respectively. Compared with the Normal control, the bodyweight of the HLP group was significantly increased. The plasma lipid levels suggested that the serum TC (Fig. 1B), TG (Fig. 1C) and LDL-C (Fig. 1D) levels in the HLP group were substantially higher than those in the Normal control group.

Histology. H\&E staining revealed that the cell nuclei of liver sections in the Normal control group were relatively large in size and were polyhedral, with round nuclei deeply stained blue. The nucleoli were clearly observed at the center of the cells. The hepatocytes were intact and tightly packed in cords, with uniform cytoplasm and without swelling or steatosis. The hepatic lobules exhibited a clear edge and normal morphology. The hepatic cords were arranged in an orderly manner displaying a radial pattern and the central vein exhibited a normal structure (Fig. 2A). By contrast, the cell morphology in the HLP group was clearly anomalous. The hepatocytes were swollen and increased in size and the cytoplasm appeared cloudy. A certain amount of inflammatory cells were observed at the periphery and lipid vacuoles of various sizes were observed in the cytoplasm of the hepatocytes. The lobule structure was damaged and vague (Fig. 2B).

Differentially expressed lncRNAs and mRNAs. A total of 96 differentially expressed mRNAs were identified from liver 
A

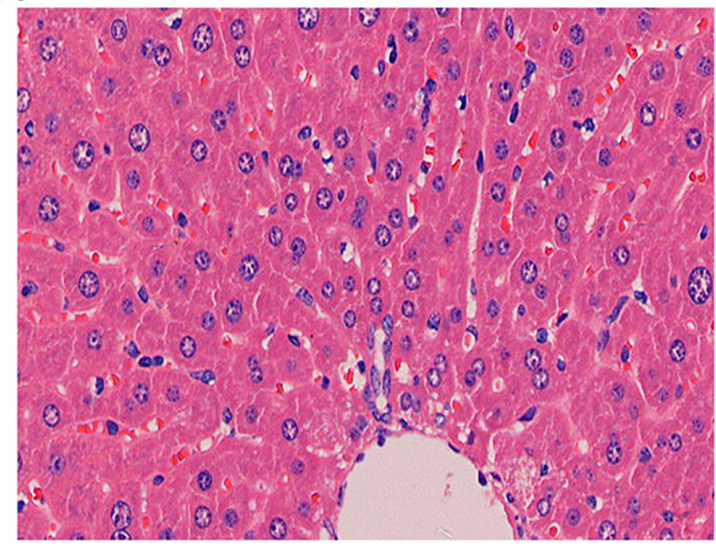

B

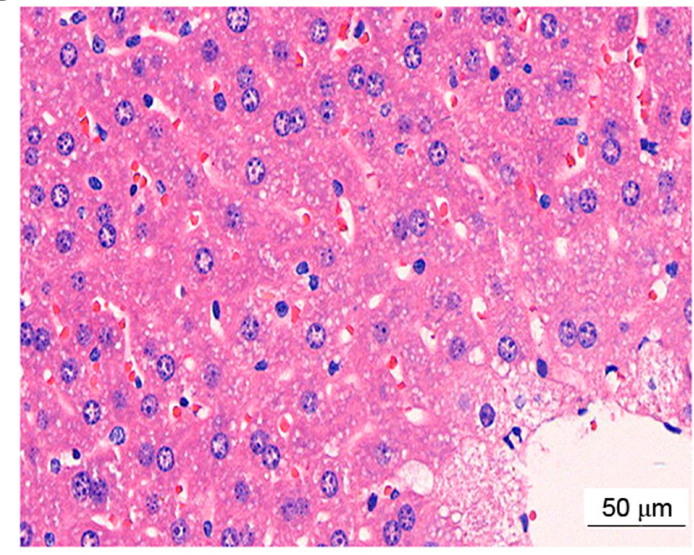

Figure 2. Histology of liver sections of mice from (A) the normal control group and (B) hyperlipidemia group [H\&E staining; original magnification, $\mathrm{x} 400$; scale bar, $50 \mu \mathrm{m}]$.

A

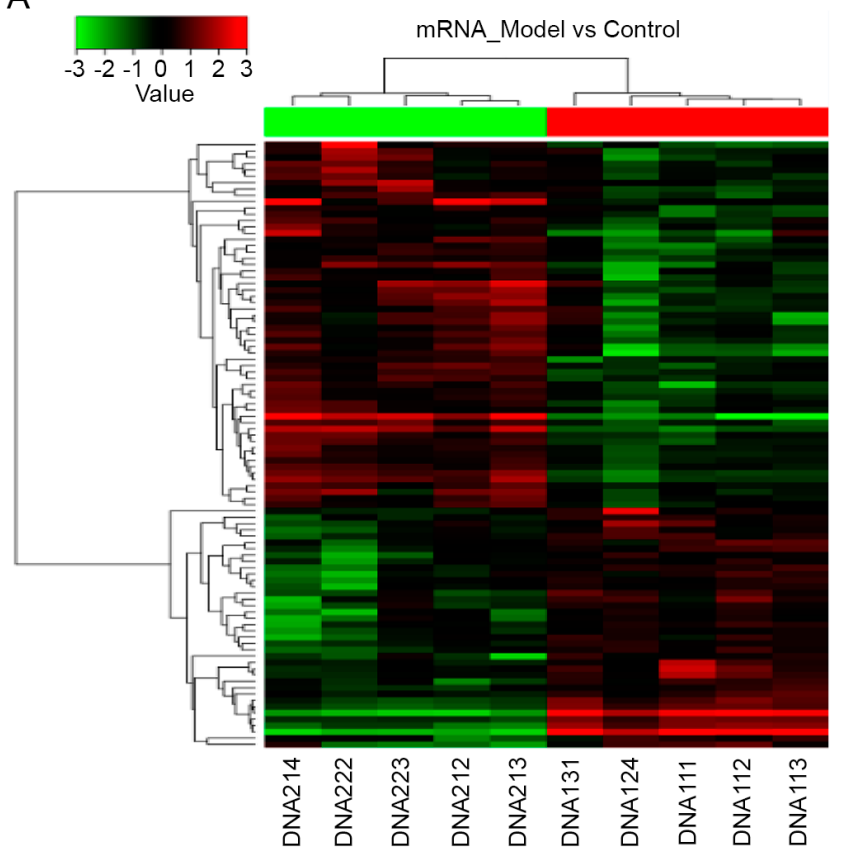

B

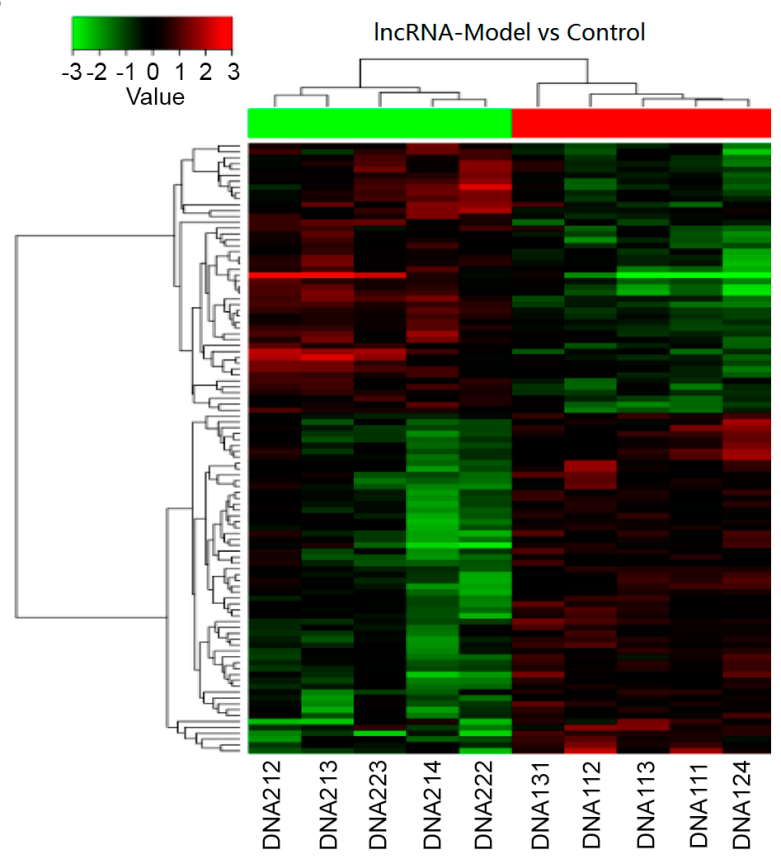

Figure 3. Heatmap of differentially expressed (A) mRNAs and (B) lncRNAs in the liver of mice with hyperlipidemia and control mice. The X-axis represents the different study groups, and the Y-axis indicates gene clusters. Red indicates upregulated genes in the liver, whereas green represents downregulated genes. lncRNA, long non-coding RNA.

samples obtained from the animals of the HLP model and Normal control groups. Compared to those in the Normal control, 58 mRNAs were upregulated and 38 were downregulated in the HLP model. Upregulated genes included those encoding carcinoembryonic antigen-related cell adhesion molecule 2 (Ceacam2), a transmembrane glycoprotein expressed in the kidney, spleen, platelets and crypt epithelial cells, and epidermal growth factor receptor (EGFR), an important transmembrane receptor implicated in multiple cancer types. In addition, 104 differentially expressed lncRNAs were identified, including 46 upregulated and 58 downregulated lncRNAs in the HLP group compared to those in the Normal control. For instance, the expression of NONMMUT008659 was upregulated, whereas that of ril0610030O19|R000004O2111299 was downregulated.
Clustering analysis of differentially expressed lncRNAs and $m R N A s$. Hierarchical clustering analysis of the DEGs between the Normal control and HLP groups was provided in a heatmap in Fig. 3. The genes belonging to the same group exhibited a relatively greater sequence similarity and the classification was clear. Red and green represent the differentially upregulated and downregulated genes in liver tissues. This clustering result may support the functional analysis of HLP-associated genes, suggesting that clustered genes are regulated together via co-expression during transcription.

Functional group analysis and GO analysis. The DEGs were analyzed for their functions and metabolic pathways using the online tool DAVID (Fig. 4). GO analysis demonstrated that the upregulated DEGs were unambiguously enriched in biological 


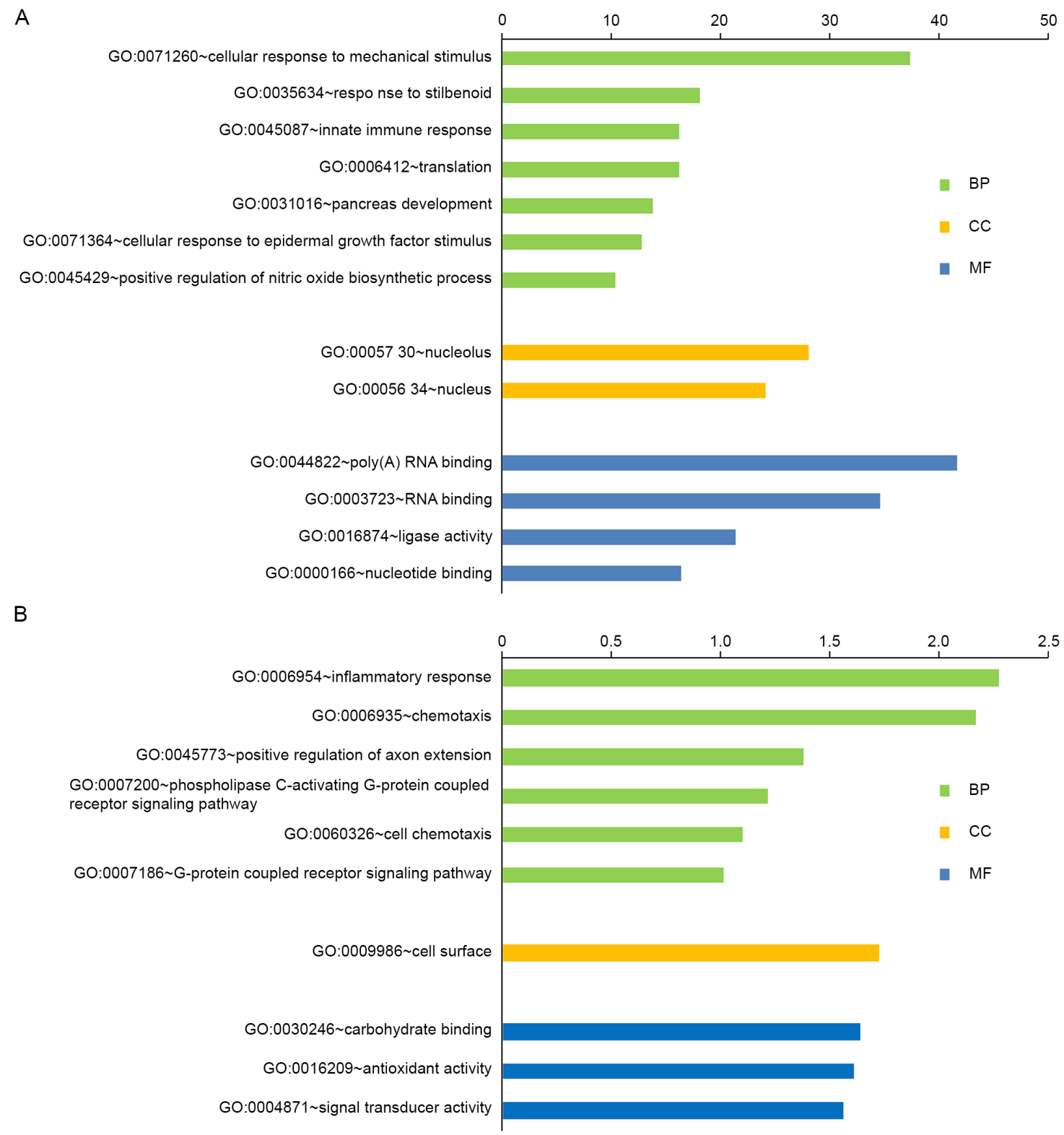

Figure 4. GO analysis of DEGs. (A) Upregulated DEGs and (B) Downregulated DEGs. Terms in the categories BP (green), CC (yellow) and MF (blue) were provided. DEG, differentially expressed gene; GO, gene ontology.

processes such as nitric oxide biosynthesis, innate immune response and pancreas development. They also had a key role as a ligase in the nucleolus and were associated with molecular functions of RNA and nucleotide binding. The downregulated DEGs were mainly associated with chemotaxis, inflammatory response and the $\mathrm{G}$ protein-coupled receptor signaling pathway. The downregulated DEGs were mainly associated with the cellular component of the cell surface. Furthermore, these genes were involved in molecular functions such as antioxidant activity, carbohydrate binding and signal transduction.

Co-expression analysis. The co-expression network included $77 \mathrm{lncRNAs}$ and their 73 related mRNAs (Fig. 5). This network consisted of 150 nodes and 451 connections, with absolute values of their correlation coefficients of $>0.9$.

Transcription factor prediction. For transcription factor prediction, gene functional annotation was performed using the DAVID database and they were aligned to the database downloaded from the transcription factor screening website http://www.tfacts.org. This led to the discovery of 7 upregulated and 4 downregulated differentially expressed mRNAs, corresponding to 60 differentially expressed lncRNAs and 38 transcription factors. Cytoscape 3.6 was used to draw the 109 nodes and lines (Fig. 6).

\section{Discussion}

Numerous studies have suggested that physiological activities such as coagulation, inflammatory response, lipid metabolism, oxidative stress and cell adhesion are closely related to the progression of HLP (15-19). However, the detailed underlying molecular mechanisms contributing to the pathogenesis of HLP have so far remained elusive. During the experiment, the body weight of the normal control group increased, but the statistical error of 


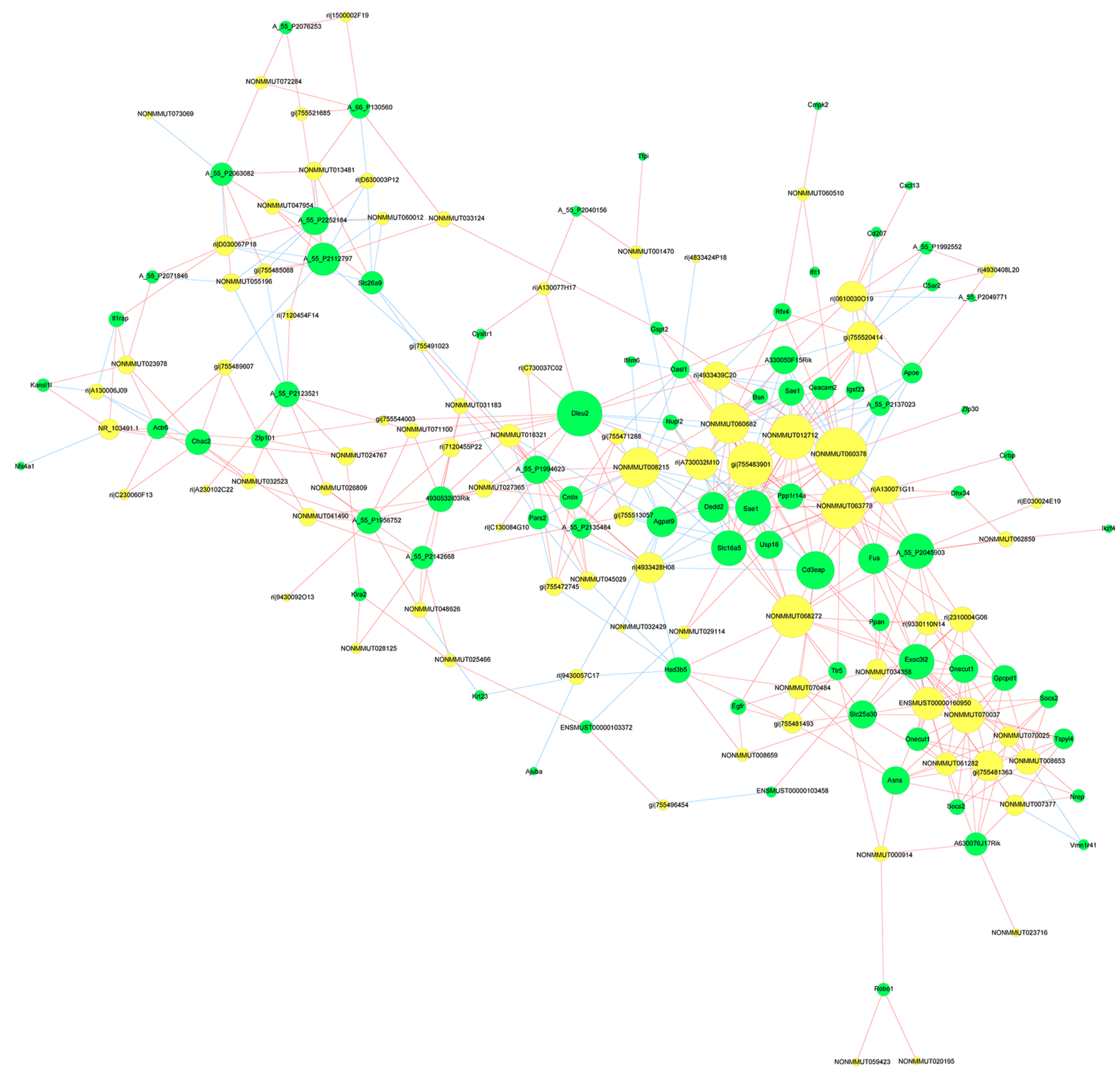

Figure 5. Co-expression analysis of differentially expressed lncRNAs and mRNAs in the hyperlipidemia mouse model. IncRNAs and mRNAs are indicated as yellow and green circles, respectively. The size of the circles represents the degree of the genes in the network (number of neighbors). Red lines denote positive correlations (345 counts), whereas blue lines indicate negative correlations (106 counts). lncRNA, long non-coding RNA.

C57BL/6J body weight due to the small sample size did not increase significantly.

In the present study, a mouse lncRNA array was used to determine the expression profile of genes in the liver of the mouse model of HLP. Caecam 2 was identified as a key upregulated gene in HLP. Caecam2 is involved in insulin secretion by regulating glucagon-like peptide-1, energy metabolism and energy expenditure, as well as brown adipogenesis, and was closely associated with metabolism in HLP. In macrophages and monocytes, the intracellular accumulation of cholesterol upregulates the expression of several responsive genes of liver $\mathrm{X}$ receptors, including ApoE, ABCA1 and ABCG1, thereby leading to a decrease in intracellular cholesterol pool and an increase in the extracellular lipid pool. In addition, EGFR emerged as a significantly upregulated gene in the HLP group. EGFR was indicated to be involved in biological processes such as cellular response to mechanical stimulus, positive regulation of nitric oxide biosynthesis, cellular response to EGF stimulus and transcription. Upon ligand stimulation, EGFR initiates intracellular signaling cascades via cytoplasmic 


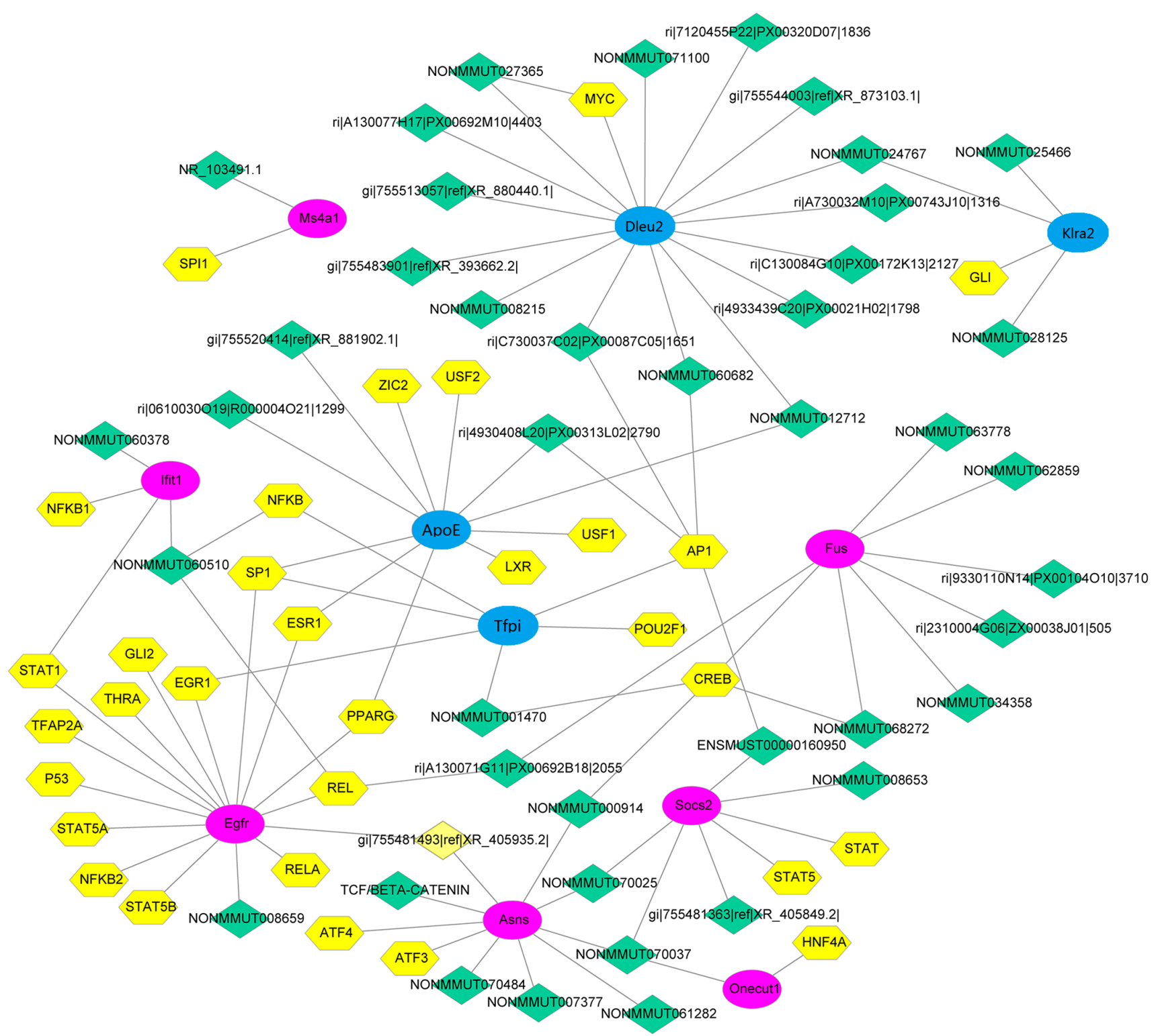

Figure 6. Transcription factor prediction. The mRNAs of DEGs are presented as ellipses, with darker pink indicating upregulated expression and lighter pink indicating downregulated expression. The green rhombuses represent the long non-coding RNAs co-expressed with the differentially expressed mRNAs. The yellow hexagons indicate transcription factors that regulate DEGs. DEG, differentially expressed gene.

adaptor proteins and enzymes, and induces cell migration, adhesion, proliferation, differentiation and apoptosis $(20,21)$.

Several genes associated with the inflammatory response were downregulated in the HLP group. These included C5AR2, CYSLTR1, CXCL13 and IL1RAP (22-25). C5AR2 and CYSLTR1 are involved in the phospholipase $\mathrm{C}$-activating GPRC signaling pathway and are strongly chemotactic $(26,27)$. C5AR2 is also a novel receptor essential for the mediation of human mast cell adhesion, migration and proinflammatory mediator production (28).

Target gene prediction revealed that AJUBA was a target gene of ril9430057C17. AJUBA is the receptor mediating the internalization of secreted glucose-regulated protein 78 into macrophages (29). In addition, it is a critical regulator of adipocyte differentiation as it functions as a specific co-activator of PPAR $\gamma$ (30). AJUBA enhances the formation of the LXR/RXR heterodimer and is vital for activation of its target gene LXR, which has an important role in regulating lipid and glucose metabolism (31). In addition, ONECUT1 was identified as the target gene of the differentially expressed lncRNA ENSMUST00000160950. ONECUT1 is a member of the Cut homeobox family of transcription factors and is expressed mainly in the liver and may be activated by growth hormone. ONECUT1 regulates the expression of genes essential for hepatic functions and is a member of hepatic transcription factors regulating the differentiation of liver functions by promoting the expression of glucose kinase and glucose-6-phosphatase involved in carbohydrate metabolism in the liver $(32,33)$.

Transcription factor prediction identified the suppressor of cytokine signaling 2 (SOCS2) and signal transducer and activator of transcription 5 (STAT5). Deletion of SOCS2 in mice was indicated to protect the liver from steatosis but aggravates insulin resistance in high-fat diet-induced mice (34). The liver of SOCS2-knockout mice displayed NF- $\mathrm{B}$ activity and impaired glucose tolerance, and an inflammatory response 
was evoked by the production of inflammatory cytokines in the liver and adipose tissues (35). Furthermore, SOCS2 mRNA levels were modulated in human hepatic steatosis (36). STAT5 is a member of the JAK/STAT signaling pathway (37). The lncRNAs NONMMUT008653, gil755481363lreflXR_405849.2I, ENSMUST00000160950 and NONMMUT070025 identified in the present study, together with SOCS2, are co-regulated by the transcription factors STAT and STAT5.

EGFR is expressed extensively in hepatocytes, controlling cell morphogenesis and/or homeostasis, including proliferation, migration and transformation (38). In addition to cell proliferation and regeneration, EGFR also exerts anti-apoptotic effects and has a regulatory role in liver and blood lipid metabolism in adult male mice (39). In a previous study, the levels of EGFR or EGFR-like receptors were positively correlated with the increase of cholesterol (40). In the present study, NONMMUT008659 and EGFR were observed to share the same transcription factors, including NF- $\kappa \mathrm{B}$ and STAT, suggesting that the pathogenesis of HLP may be associated with the co-regulation of these transcription factors.

ApoE is a multifunctional plasma glycoprotein $(34 \mathrm{kDa})$ secreted by macrophages and other immune cells in several tissues, including the liver, brain, kidney, adrenal gland and adipose tissue, and is a key component that mediates the binding of all lipoproteins. A genome-wide association study indicated that ApoE and LDL-C levels were tightly coupled (41). ApoE participates in the destabilization of the $\alpha$-helix in the binding domain and suppresses the degradation of LDL. Furthermore, mutant ApoE binds to LDL receptor and disrupts efficient recycling of LDLR to the hepatocyte surface. Thus, HLP is closely associated with ApoE protein levels. In the present study, ApoE expression was downregulated in the liver, with transcription factors SP1 and ESR1 co-regulating the expression of ApoE and EGFR. These results suggested that the IncRNAs gil755520414|reflXR_881902.1I and ril0610030O19IR000004021/1299 may be engaged in the regulation of ApoE expression via EGFR and SP1 to impact plasma lipid metabolism. Hepatocyte nuclear factors (HNFs), namely HNF4A, HNF4C, ONECUT1 and Onecut 2 , are expressed in a variety of organs and tissue types, including the liver, pancreas and kidney. As mentioned above, ONECUT1 regulates glucose metabolism and the cell cycle (42). The present results revealed that ONECUT1 and HNF4A were regulated by NONMMUT070037, which controlled both SOCS2 and ASNS, in the HLP group.

In summary, in the present study, 104 differentially expressed lncRNAs were identified in the mouse model of HLP compared to the Normal control animals. They included 46 upregulated (e.g., ENSMUST00000160950) and 58 downregulated (e.g., ril9430057C17) lncRNAs. In addition, 96 differentially expressed mRNAs were identified, including 58 upregulated genes (e.g., EGFR and SOCS2) and 38 downregulated genes (e.g., C5AR2, APOE, CYSLTR1 and CXCL13). These results strongly indicate the significance of DEGs in the pathogenesis of HLP. In particular, ENSMUST00000160950, EGFR, SOCS2 and ApoE may be potential therapeutic targets for the clinical management of HLP. However, further studies are warranted to evaluate this hypothesis toward clinical implications.

\section{Acknowledgements}

The authors would like to thank Professor Yulong Chen and Professor Hui Wang of the College of Traditional Chinese Medicine, Henan University of Traditional Chinese Medicine (Zhengzhou, China) for their help with the animal experiments and project design.

\section{Funding}

This study was supported by a grant from the study on the distribution rule of traditional Chinese medicine constitution in patients with carotid atherosclerotic plaque (grant no. 20140518).

\section{Availability of data and materials}

The datasets used and/or analyzed during the current study are available from the corresponding author upon reasonable request. The microarray data of the present study were deposited in the Gene Expression Omnibus (GEO) database (http:// www.ncbi.nlm.nih.gov/geo/; GEO accession no. GSE169398).

\section{Authors' contributions}

YC designed and conceived the study. BX performed data analysis and literature collection. NW wrote the manuscript and performed the bioinformatics analysis. XX drew the charts and performed statistical processing of the data. All authors have read and approved the final manuscript.

\section{Ethics approval and consent to participate}

The present study was approved by the Ethics Committee of Henan University of Chinese Medicine (ethics review approval no. DWLL201704101).

\section{Patient consent for publication}

Not applicable.

\section{Competing interests}

The authors declare that they have no competing interests.

\section{References}

1. Ried K, Toben C and Fakler P: Effect of garlic on serum lipids: An updated meta-analysis. Nutr Rev 71: 282-299, 2013.

2. Tosheska Trajkovska K and Topuzovska S: High-density lipoprotein metabolism and reverse cholesterol transport: Strategies for raising HDL cholesterol. Anatol J Cardiol 18: 149-154, 2017.

3. Hassing HC, Surendran RP, Derudas B, Verrijken A, Francque SM, Mooij HL, Bernelot Moens SJ, Hart LM, Nijpels G, Dekker JM, et al: SULF2 strongly prediposes to fasting and postprandial triglycerides in patients with obesity and type 2 diabetes mellitus. Obesity (Silver Spring) 22: 1309-1316, 2014.

4. Walther TC and Farese RV Jr: Lipid droplets and cellular lipid metabolism. Annu Rev Biochem 81: 687-714, 2012.

5. Endo H, Shiroki T, Nakagawa T, Yokoyama M, Tamai K, Yamanami H, Fujiya T, Sato I, Yamaguchi K, Tanaka N, et al: Enhanced expression of long non-coding RNA HOTAIR is associated with the development of gastric cancer. PLoS One 8: e77070, 2013 
6. Hirose T, Mishima Y and Tomari Y: Elements and machinery of non-coding RNAs: Toward their taxonomy. EMBO Rep 15 489-507, 2014

7. Lee JT: Epigenetic regulation by long noncoding RNAs. Science 338: 1435-1439, 2012.

8. Zhang Y, Sun L, Xuan L, Pan Z, Hu X, Liu H, Bai Y, Jiao L, Li Z, Cui L, et al: Long non-coding RNA CCRR controls cardiac conduction via regulating intercellular coupling. Nat Commun 9 : 4176, 2018.

9. Uchida S and Dimmeler S: Long noncoding RNAs in cardiovascular diseases. Circ Res 116: 737-750, 2015.

10. Chen J, Cui X, Shi C, Chen L, Yang L, Pang L, Zhang J, Guo X, Wang J and Ji C: Differential lncRNA expression profiles in brown and white adipose tissues. Mol Genet Genomics 290: 699-707, 2015.

11. Smekalova EM, Kotelevtsev YV, Leboeuf D, Shcherbinina EY, Fefilova AS, Zatsepin TS and Koteliansky V: IncRNA in the liver: Prospects for fundamental research and therapy by RNA interference. Biochimie 131: 159-172, 2016.

12. Han P, Li W, Lin CH, Yang J, Shang C, Nuernberg ST, Jin KK, $\mathrm{Xu} \mathrm{W}, \mathrm{Lin} \mathrm{CY}$, Lin CJ, et al: A long noncoding RNA protects the heart from pathological hypertrophy. Nature 514: 102-106, 2014.

13. Guo W, Zhang H, Yang A, Ma P, Sun L, Deng M, Mao C, Xiong J, Sun J, Wang N, et al: Homocysteine accelerates atherosclerosis by inhibiting scavenger receptor class B memberl via DNMT3b/ SP1 pathway. J Mol Cell Cardiol 138: 34-48, 2020.

14. Leary S, Underwood W and Anthony R: AVMA Guidelines for the Euthanasia of Animals: 2013 Edition. 2013. American Veterinary Medical Association. Available from: avma.org/sites/ default/files/2020-01/2020-Euthanasia-Final-1-17-20.pdf.

15. Ozay R, Uzar E, Aktas A, Uyar ME, Gürer B, Evliyaoglu O, Cetinalp NE and Turkay C: The role of oxidative stress and inflammatory response in high-fat diet induced peripheral neuropathy. J Chem Neuroanat 55: 51-57, 2014.

16. Owens AP III, Byrnes JR and Mackman N: Hyperlipidemia, tissue factor, coagulation, and simvastatin. Trends Cardiovasc Med 24: 95-98, 2014.

17. Liu TT, Zeng Y, Tang K, Chen X, Zhang W and Xu XL: Dihydromyricetin ameliorates atherosclerosis in LDL receptor deficient mice. Atherosclerosis 262: 39-50, 2017

18. Xenoulis PG and Steiner JM: Lipid metabolism and hyperlipidemia in dogs. Vet J 183: 12-21, 2010.

19. Lee YJ, Seo JA, Yoon T, Seo I, Lee JH, Im D, Lee JH, Bahn KN, Ham HS, Jeong SA, et al: Effects of low-fat milk consumption on metabolic and atherogenic biomarkers in Korean adults with the metabolic syndrome: A randomised controlled trial. J Hum Nutr Diet 29: 477-486, 2016.

20. Singhai A, Wakefield DL, Bryant KL, Hammes SR, Holowka D and Baird B: Spatially defined EGF receptor activation reveals an F-actin-dependent phospho-Erk signaling complex. Biophys J 107: 2639-2651, 2014.

21. Zaczek A, Brandt B and Bielawski KP: The diverse signaling network of EGFR, HER2, HER3 and HER4 tyrosine kinase receptors and the consequences for therapeutic approaches. Histol Histopathol 20: 1005-1015, 2005.

22. Coulthard LG, Hawksworth OA and Woodruff TM: Complement: The emerging architect of the developing brain. Trends Neurosci 41: 373-384, 2018.

23. Matsumoto H, Kanemitsu Y, Nagasaki T, Tohda Y, Horiguchi T, Kita H, Kuwabara K, Tomii K, Otsuka K, Fujimura M, et al Staphylococcus aureus enterotoxin sensitization involvement and its association with the CysLTR1 variant in different asthma phenotypes. Ann Allergy Asthma Immunol 118: 197-203, 2017.

24. Kowarik MC, Cepok S, Sellner J, Grummel V, Weber MS, Korn T, Berthele A and Hemmer B: CXCL13 is the major determinant for B cell recruitment to the CSF during neuroinflammation. J Neuroinflammation 9: 93, 2012.

25. Askmyr M, Ågerstam H, Hansen N, Gordon S, Arvanitakis A Rissler M, Juliusson $G$, Richter $J$, Järås $M$ and Fioretos T: Selective killing of candidate AML stem cells by antibody targeting of IL1RAP. Blood 121: 3709-3713, 2013.
26. Hu C, Yang J, He Q, Luo Y, Chen Z, Yang L, Yi H, Li H, Xia H, Ran D, et al: CysLTR1 blockage ameliorates liver injury caused by aluminum-overload via PI3K/AKT/mTOR-mediated autophagy activation in vivo and in vitro. Mol Pharm 15: 1996-2006, 2018.

27. Kistler B, Schlegel A, Grauert M, Arndt K and Williams C: Cell type specific accessibility of C5aR2 on human blood cells of myeloid origin. Eur Resp J 48: PA3637, 2016.

28. Pundir P, MacDonald CA and Kulka M: The novel receptor $\mathrm{C} 5 \mathrm{aR} 2$ is required for C5a-mediated human mast cell adhesion, migration, and proinflammatory mediator production. J Immunol 195: 2774-2787, 2015.

29. La X, Zhang L, Li H, Li Z, Song G, Yang P and Yang Y: Ajuba receptor mediates the internalization of tumor-secreted GRP78 into macrophages through different endocytosis pathways. Oncotarget 9: 15464-15479, 2018.

30. Li Q, Peng H, Fan H, Zou X, Liu Q, Zhang Y, Xu H, Chu Y, Wang C, Ayyanathan K, et al: The LIM protein Ajuba promotes adipogenesis by enhancing PPAR $\gamma$ and $\mathrm{p} 300 / \mathrm{CBP}$ interaction. Cell Death Differ 23: 158-168, 2016.

31. Liu QY, Quinet E and Nambi P: Adipocyte fatty acid-binding protein (aP2), a newly identified LXR target gene, is induced by LXR agonists in human THP-1 cells. Mol Cell Biochem 302: 203-213, 2007.

32. Beaudry JB, Pierreux CE, Hayhurst GP, Plumb-Rudewiez N, Weiss MC, Rousseau GG and Lemaigre FP: Threshold levels of hepatocyte nuclear factor 6 (HNF-6) acting in synergy with HNF-4 and PGC-1alpha are required for time-specific gene expression during liver development. Mol Cell Biol 26: 6037-6046, 2006.

33. Holterman A, Wang K, Wang M and Gannon M: Transcriptional regulation of hepatic genes by hepatocyte nuclear factor $\mathrm{OC} 1$ attenuates liver injury. J Surg Res 158: 324, 2010.

34. Zadjali F, Santana-Farre R, Vesterlund M, Carow B, Mirecki-Garrido M, Hernandez-Hernandez I, FlodströmTullberg M, Parini P, Rottenberg M, Norstedt G, et al: SOCS2 deletion protects against hepatic steatosis but worsens insulin resistance in high-fat-diet-fed mice. FASEB J 26: 3282-3291, 2012.

35. Leroith D and Nissley P: Knock your SOCS off! J Clin Invest 115: 233-236, 2005.

36. Vesterlund M: The mechanism of action of SOCS2 and its role in metabolism and growth. Arthritis Rheum 60: 1743-1752, 2013

37. Terrell AM, Crisostomo PR, Wairiuko GM, Wang M, Morrell ED and Meldrum DR: Jak/STAT/SOCS signaling circuits and associated cytokine-mediated inflammation and hypertrophy in the heart. Shock 26: 226-234, 2006.

38. Komposch K and Sibilia M: EGFR signaling in liver diseases. Int J Mol Sci 17: 30, 2015.

39. Scheving LA, Zhang X, Garcia OA, Wang RF, Stevenson MC, Threadgill DW and Russell WE: Epidermal growth factor receptor plays a role in the regulation of liver and plasma lipid levels in adult male mice. Am J Physiol Gastrointest Liver Physiol 306: G370-G381, 2014.

40. Leedman P, Giles K and Webster RJ: Method of modulation of expression of epidermal growth factor receptor (EGFR) involving miRNA. Patent application EP, US86738722014. Filed 28 August 2007; issued 6 March, 2008.

41. Deshmukh HA, Colhoun HM, Johnson T, McKeigue PM, Betteridge DJ, Durrington PN, Fuller JH, Livingstone S, Charlton-Menys V, Neil A, et al: Genome-wide association study of genetic determinants of LDL-c response to atorvastatin therapy: Importance of Lp(a). J Lipid Res 53: 1000-1011, 2012.

42. Yamamoto K, Matsuoka T, Kawashima S, Takebe S, Kubo F, Miyatsuka T, et al: A novel function of Onecut1 as a negative regulator of MafA. J Biol Chem 288: 21648-21658, 2013.

This work is licensed under a Creative Commons Attribution-NonCommercial-NoDerivatives 4.0 International (CC BY-NC-ND 4.0) License. 